\title{
Production and Quality Control of the Scintillator Surface Detector for the AugerPrime Upgrade of the Pierre Auger Observatory
}

\author{
Jan Pękala ${ }^{* a}$ for the Pierre Auger Collaboration ${ }^{b \dagger}$ \\ ${ }^{a}$ Institute of Nuclear Physics PAS, Radzikowskiego 152, 31-342 Kraków, Poland \\ ${ }^{b}$ Observatorio Pierre Auger, Av. San Martín Norte 304, 5613 Malargüe, Argentina \\ E-mail: auger_spokespersonsefnal.gov \\ Full author list: http://www.auger.org/archive/authors_icrc_2019.html
}

\begin{abstract}
The Pierre Auger Observatory is undergoing a major upgrade, called AugerPrime. One of the goals of this upgrade is to improve the capabilities of the existing stations of the surface detector. The main modification consists in the addition of a new scintillator surface detector (SSD) to each water-Cherenkov detector station. The large-scale production of the SSDs started in 2017. The detector assembly is done in six laboratories with the collaboration of many other institutions providing components. Each institution involved follows common procedures, making sure that all the high standards set by the design are being met. We describe these procedures, which include quality checks of all components and a final certification of each detector with cosmicray muons. The deployment of the SSDs at the Observatory is already under way.
\end{abstract}

36th International Cosmic Ray Conference -ICRC2019-

July 24th - August 1st, 2019

Madison, WI, U.S.A.

\footnotetext{
* Speaker.

${ }^{\dagger}$ for collaboration list see PoS(ICRC2019)1177
} 


\section{Introduction}

The Pierre Auger Observatory [1] is currently undergoing a major upgrade, called AugerPrime $[2,3]$. The main goal of this upgrade is to better determine the cosmic-ray composition at the highest energies. The composition has already been studied using the hybrid method of air shower observation, i.e., simultaneously by the surface detector (SD) and the fluorescence detector (FD) [4]. However, the limited duty cycle of the FD translates into low event statistics above $\sim 10^{19.5} \mathrm{eV}$. The new AugerPrime detector arrangement will result in composition-sensitive data in this interesting region of flux suppression.

The main element of the AugerPrime upgrade is the addition of new scintillator surface detectors (SSDs) on top of the existing water-Cherenkov detectors (WCDs) of the SD. The SSDs will provide complementary measurements of air-shower particles arriving at the ground. Scintillator detectors have responses to muons and electromagnetic particles that are different from those of the WCDs, which means that this additional information will enable a reliable disentangling of these components of air showers.

Further elements of the upgrade include: the addition of a fourth small (1" diameter) photomultiplier tube (PMT) to the WCDs; equipping the SD stations with new electronics [5]; augmenting the duty cycle of the FD by about 50\%; an extension of the AMIGA (Auger Muons and Infill for the Ground Array) project by an additional array of 61 scintillator detectors buried $2.3 \mathrm{~m}$ underground [6]; and adding radio detectors to every SD station [7].

\section{Design of the SSD}

The SSD was designed to be large enough to measure the density of air-shower particles with statistical uncertainties comparable to those of the WCDs. On the other hand, the requirement that the SSD be mounted on top of the WCD tank placed upper limits on the size and the weight of the SSD. The detector must be resistant and durable, to withstand several years of exposure to different weather conditions at the Pierre Auger Observatory, at locations where maintenance is very difficult. The cost of the SSDs was also an important factor.

The active part of the SSD is comprised of scintillator bars of dimensions $160 \mathrm{~cm} \times 5 \mathrm{~cm}$ $\times 1 \mathrm{~cm}$, produced on the FNAL/NICADD Extrusion Line [8]. Each scintillator has two coextruded channels through which wavelength-shifting optical fibers are routed, and an outer protective/reflective layer of $\mathrm{TiO}_{2}$. There are 48 scintillators in each SSD, placed symmetrically on both sides of the detector (fig. 1 left).

Plastic wavelength-shifting fibers are used to collect the light emitted inside the scintillators. A Kuraray Y11(300)M S-type fiber, of $1 \mathrm{~mm}$ diameter, was chosen, as its absorption spectrum matches well that of the light produced in the scintillators. Each fiber runs inside two scintillator bars through channels separated by a distance of $10 \mathrm{~cm}$, with both ends meeting in the central part of the SSD. Outside the scintillators the fibers are guided in styrofoam routers.

All 96 fiber ends are bundled into a "cookie" - a poly(methyl methacrylate) (PMMA) cylinder that provides optical coupling to a single PMT. The cookie is fixed to an aluminum tube placed in the middle of one of the long sides of the SSD frame. The tube serves as a housing for a 

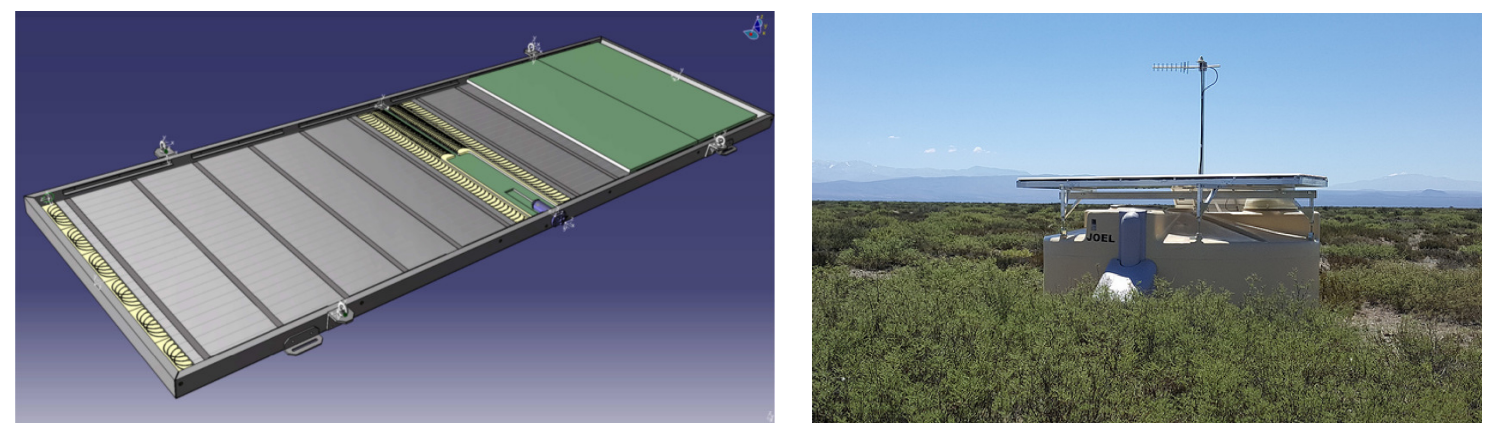

Figure 1: Drawing of an open SSD, showing the layout of the components (left), and photo of one of the deployed SSDs (right).

Hamamatsu R9420 PMT (1.5" diameter). The PMT can be installed in a completed SSD at any later time, during the deployment of the detectors.

The main mechanical element of the SSD is its enclosure box [9]. The sides of the rectangular box $(3.8 \mathrm{~m} \times 1.3 \mathrm{~m})$ are made of aluminum profiles. A composite panel, made of two sheets of aluminum glued to a block of extruded polystyrene, serves as the bottom of the box; it strengthens the structure, and also serves as a support for all of the inner components of the detector. On top the box is closed with an aluminum sheet. All elements of the enclosure box are glued together using Ottocoll S610 silicone sealant, which ensures that the box is light-tight. To allow for equalization of pressure, several small holes are drilled into the frame; to keep the enclosure light-tight the inner hole is covered (glued and riveted) with a disk of sintered metal.

Additionally on top of the box a sunroof is installed: corrugated sheets reflecting sunlight and allowing for air flow, which prevents excessive heating. The completed detector is mounted on top of the WCD tank using a rigid support frame, built of aluminum beams.

The design of the SSD was tested and validated in the Pierre Auger Observatory. Twelve prototype detectors were deployed in late 2016, and since then the upgraded stations have been continuously in operation [10].

\section{Organization of the production}

The AugerPrime upgrade is a joint effort of all institutions of the Pierre Auger Collaboration. Numerous institutions take part in working on different elements of the upgrade. This joint work is especially prominent in the building of the SSDs, where all institutions involved collaborate closely. Many of the components were purchased or manufactured by one institution, and then distributed among partners according to demand. The remaining components and materials were procured locally by each institution - aluminum profiles for the frames were ordered from commercial manufacturers, and small mechanical parts were bought or produced in local workshops. The assembly of the detectors has been distributed among six sites: RWTH Aachen University, Laboratory of Subatomic Physics and Cosmology Grenoble, Karlsruhe Institute of Technology, Institute of Nuclear Physics Kraków, INFN Lecce and NIKHEF Nijmegen, with two more institutions working on PMT preparations: Bergische Universität Wuppertal and INFN Napoli. 

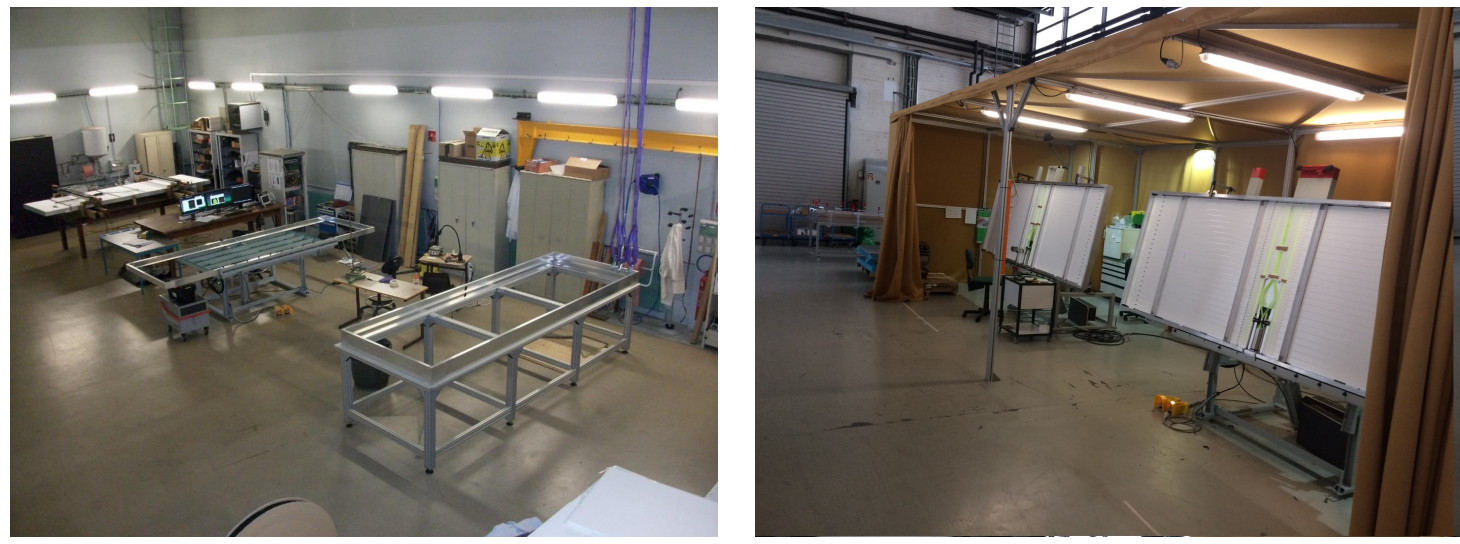

Figure 2: Workshops dedicated to SSD assembly in Grenoble (left) and Karlsruhe (right).

Each of the institutions has been able to assemble the SSDs thanks to the resources already available there, e.g., technical departments experienced in building scientific instruments. Some adaptations were necessary, like isolation of any particular SSD assembly area, to protect the fibers and scintillators from UV light exposure (fig. 2). Dedicated equipment had to be prepared prior to the assembly process, like tiltable tables, at which gluing of fibers into cookies takes place. Also test measurement stations had to be prepared. Having built the SSD prototypes, the Karlsruhe group hosted staff from other sites to share their experience, and also visited them at their institutions when the SSD assembly was starting.

During the preparations and early stages of production, all problems encountered, as well as any minor non-conformities were reported and discussed, and solutions to deal with them formulated. As a result of this process, a detailed assembly procedure was developed. All separate fabrication and assembly sites follow this common procedure, which ensures that all of the SSD design standards are met.

\section{Assembly procedure}

The assembly of a SSD starts with preparation of the frame of the enclosure box. Four pieces of aluminum profile, with ends cut at a $45^{\circ}$ angle, are glued into a rectangular frame using insert corner pieces and Ottocoll S610 sealant. Alternatively, frames welded by a commercial provider were used in Krakow and Grenoble. Next a composite panel is glued into the frame, using the same sealant that ensures light-tightness. After each step the glue is allowed to cure for a day. Before assembling the detector, a number of holes are cut in the frame to accommodate the aluminum tube of the PMT housing (fig. 3 right - bottom part), to allow for equalization of pressure, and for rivets used to attach support brackets on the outside and U-beams fixing the scintillators on the inside.

Prior to assembly, the ends of the channels in the scintillators are graded, to remove any rough edges, and compressed air is blown through the channels, to make sure they are clean. Outer routers are placed at both ends of the SSD frame, and then the scintillators are positioned, 24 bars on each side. Finally, inner routers are placed and fixed at the inner ends of the scintillators. Next, the fibers are inserted - usually directly from the spools on which they are delivered (fig. 3 left). Each fiber is inserted into one scintillator bar, then at the other end it is guided following a groove in the router 


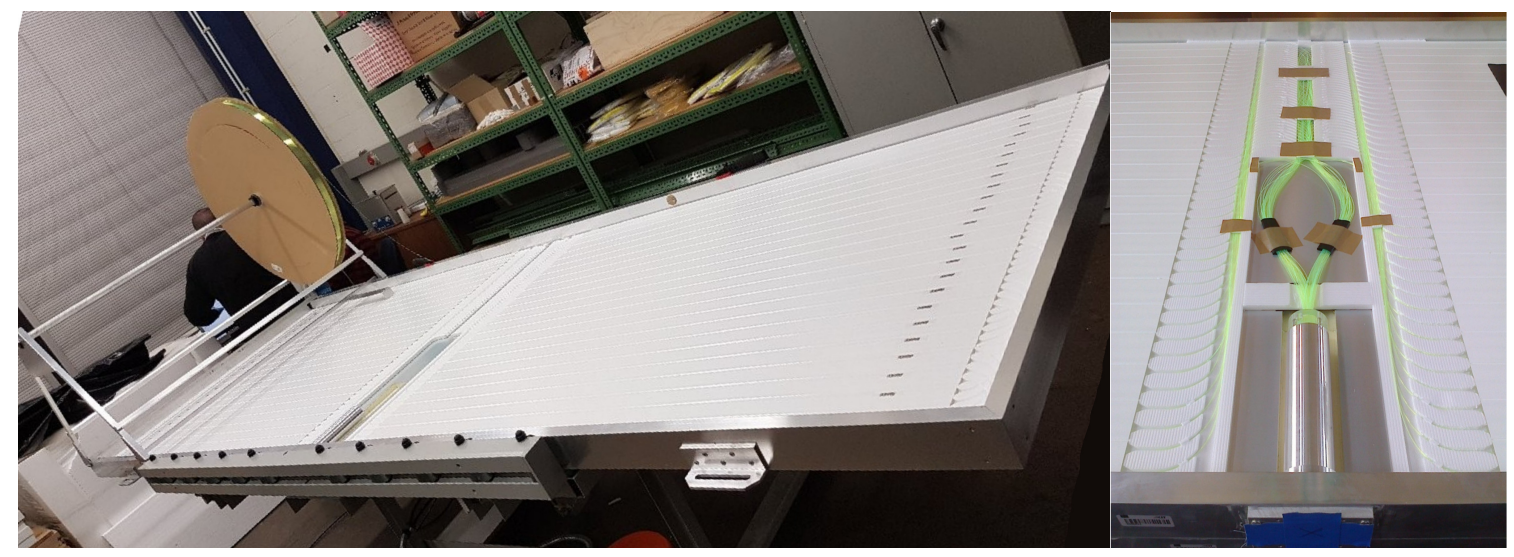

Figure 3: Inserting fibers into scintillators (left) and the inner part of an assembled SSD (right).

to be inserted into another bar. The remaining parts of the fiber are fixed in the proper grooves of the inner routers, making sure that both ends are of the same length.

The next steps in the assembly process are carried out at tiltable tables raised to the upright position (fig. 2 right), which allows for easier access and facilitates greatly the optical gluing of the 96 fiber ends inside the cookie. Fiber ends require finishing after cutting, to prevent inefficiency in light collection. As an alternative to more difficult polishing, the plastic fiber ends are melted. A borosilicate glass plate heated to $150 \pm 30^{\circ} \mathrm{C}$ is used. Each fiber end is delicately placed on this plate for about one second, which is sufficient to melt and flatten the cut surface of the fiber (fig. 4 left). The improvement of the light transmission is directly visible in a reflection off the heated plate. In case the fiber gets melted for too long, a bead may appear, which can be easily corrected by cutting off this small piece of fiber and repeated melting.

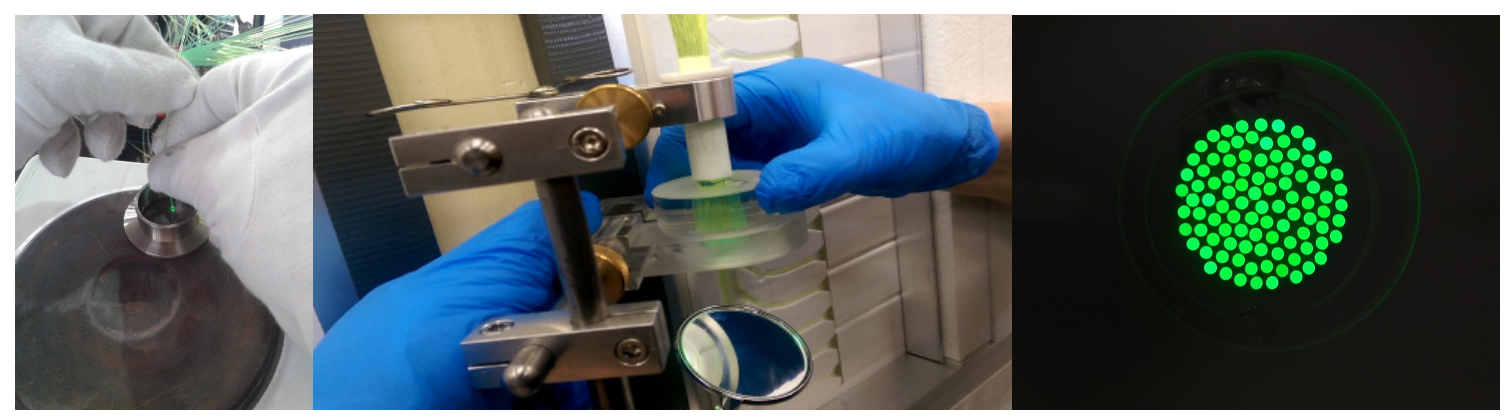

Figure 4: Fiber ends are melted in order to get a flat, smooth surface (left) and are glued inside the cookie (center). A photograph of a ready cookie (right).

All 96 fiber ends are bundled and placed in the opening of the PMMA cookie. The front surfaces of the fibers are aligned on the surface of the cookie window, which is then moved away by about $1 \mathrm{~mm}$, to leave some space for the optical glue. Eljen EJ-500 is used as the optical cement. As a two-component glue, it requires mixing, after which it needs to be set aside or dessicated to get rid of air bubbles trapped during the mixing. The glue is poured slowly, to allow all air to escape through the hole designed for this purpose. In case an air bubble gets caught in front of the fiber ends, it is still possible to force it out by twisting or tapping the cookie (fig. 4 center). After 

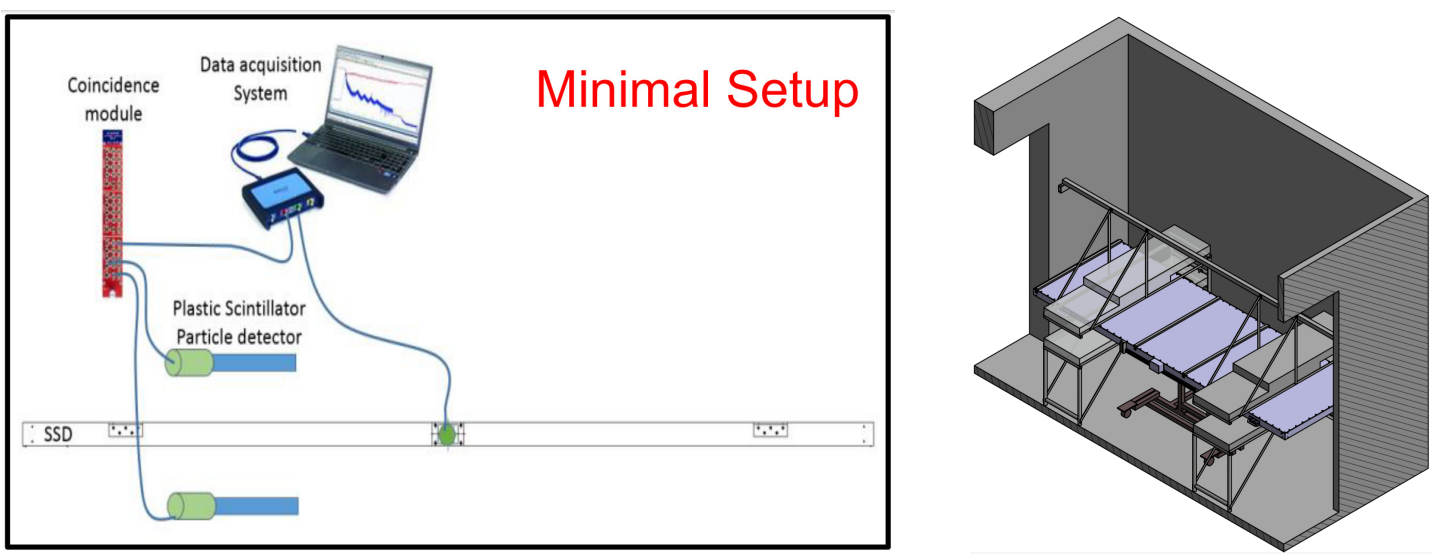

Figure 5: Scheme of a minimal setup for local tests of SSDs (left) and drawing of the test stand in Nijmegen (right).

allowing sufficient time for the glue to cure (usually overnight), the cookie is fixed to the inner end of the aluminum tube (fig. 3 right and fig. 4 right).

To minimize the enclosed volume of air inside the SSD and to additionally fix the components, the remaining volume of the frame is filled with lightweight styrofoam blocks. Next, the detector is closed by gluing and riveting the top cover. Finally, the roof support bars are attached. The sunroof itself is mounted later, prior to deployment at the Observatory.

The procedure for assembling all SSD units includes quality checks at each step. All components are inspected before they are used. Especially important is ensuring the quality of those components of the SSD that produce and guide light: scintillators, fibers and cookie. The greatest risk of damage occurs during the insertion of the fibers into the scintillators. The fiber can occasionally but rarely get scratched or bent too much, even with trained and experienced personnel. In such a case it gets replaced with a new piece. Finishing the fiber ends and gluing the cookie is done by the most experienced staff members. Every step is controlled and documented, and the information about all components used to build all detectors is recorded and stored in a database especially developed for this purpose (e.g. SSD serial number, date of production, individual bar-codes of the scintillators, batch numbers of fibers and glues, picture of the fiber ends glued in the cookie).

\section{Validation of the detectors}

In addition to the controls at each step of the assembly, all SSDs undergo additional tests at each site upon completion. These tests provide an opportunity to detect any deficiencies that could not be identified earlier, as well as enabling a baselining of the quality of data each detector can provide. Each of the SSD production sites has at least one PMT at its disposal, with which it is possible to test the SSDs.

The tests are done with cosmic-ray muons. This enables us to determine the detector response to a reference minimum-ionizing particle (MIP). To identify these particles it is necessary to have an external trigger system. At two SSD production sites (Karlsruhe and Lecce) it was possible to use already existing muon detectors, that provide a trigger over the whole area of the SSD, and a high-resolution $(\sim 1 \mathrm{~cm})$ measurement of the muon track. At other sites dedicated test stations were 

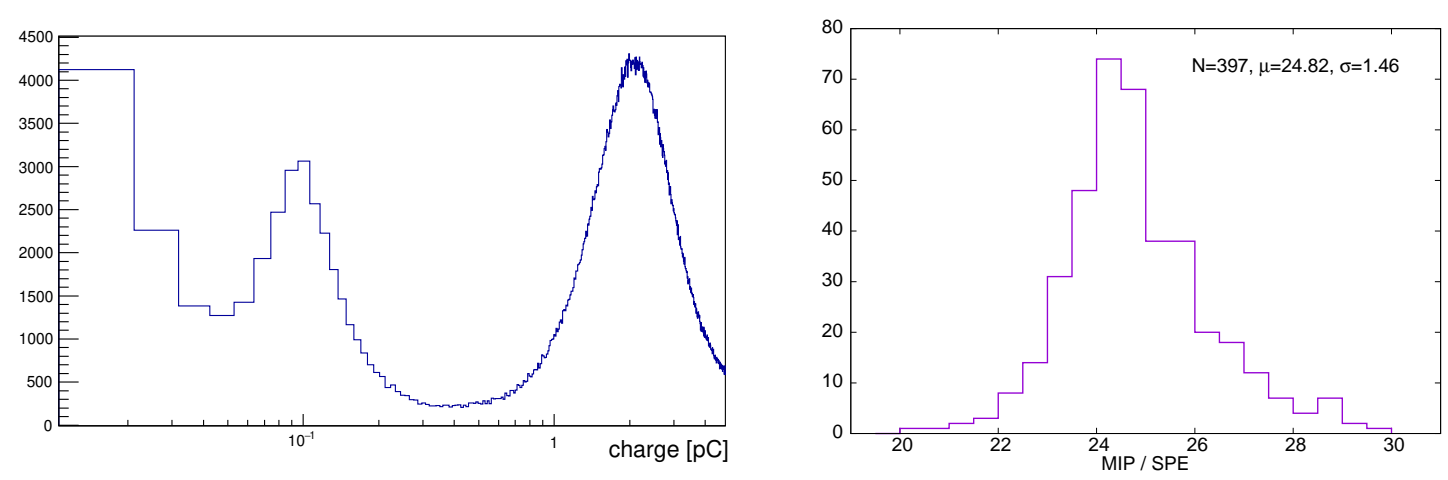

Figure 6: Histogram of signals recorded in a test measurement, with peaks corresponding to electronics noise, SPEs and MIPs (left). Distribution of test results for SSDs made in Karlsruhe (right).

prepared, using components available locally. A minimum requirement was to have two particle detectors working in coincidence, that would provide a trigger over a fraction of the SSD active area (fig. 5 left). The measurement should cover as large and as representative a part of the SSD area as possible. This was done by building the external detectors so they cover a fraction of all scintillators, on both sides of the SSD, during a measurement (fig. 5 right), or alternatively by moving smaller detectors over different parts of the SSD in the course of a test run. Each test measurement takes at least a few hours, to collect a sufficient number of MIP events.

The test measurements allow us to identify not only signals of MIPs, but also those of single photoelectrons (SPE). Fits of the peaks in the histograms are used to determine the mean charge of the MIP and SPE (fig. 6 left). The ratio of these values can be used as an estimate of the quality of the tested SSD - it reflects the efficiency of the key detector components at generating (scintillators), collecting (fibers) and transmitting (cookie) the light. An example of test results from one institution is shown in fig. 6 (right). Results from different institutions are obtained using different test setups (including different PMTs). To enable some comparison, 2 or 3 detectors from each production site were sent for test measurements to Karlsruhe. These cross-tests are used to rescale the results obtained in other institutions, which show that they are in good agreement, with mean values of MIP/SPE $\sim 24 \pm 2$, i.e. exceeding the design requirements by a factor of $\sim 2$.

The total efficiency of SSDs is measured in testing, as well as the uniformity of response over the active area of the detector. Institutions that had the advantage of high-resolution detectors performed detailed studies of some detectors. These showed that signals from individual scintillator bars deviate typically by no more than $10 \%$ from the average. Signals from opposing ends of a scintillator bar differ by $\sim 5 \%$, as follows from the light attenuation in the fiber. For routine measurements at all sites the shape of the MIP peak in the histogram of all recorded signals can be used as an estimate of detector uniformity. If a significant part of the detector were deficient, it would cause a deformation of this peak. A Gaussian fit sigma of the order of $30 \%$ of the mean value is a sign of a satisfactory uniformity of the tested SSDs.

Another test is done by measuring the trigger rate of each SSD. Any significant deviation from expected trigger rates (which should be stable for a fixed electronics setup) would be an indication of some problem with a given SSD. An increase of trigger frequency is an indication that the 
enclosure box is not light-tight. Even careful visual inspection during and after gluing might not be enough to ensure complete light-tightness, and this test helps to verify this - to make any light leaks more prominent, a high intensity light is directed in the course of the test at all glued interfaces. A trigger rate smaller than expected could be caused by some damage in a part of the active area of the SSD or a light loss in the cookie. All test measurements are also stored in the SSD database, including e.g. values of MIP and SPE, histograms of measured signals, dark count rate.

\section{Status of the production and deployment}

The large-scale production of the SSDs started in 2017. As of the middle of 2019 the production of the SSDs is nearing its end, with some of the sites having already finished their share. The completed detectors are being shipped in batches to Argentina. The SSDs have been undergoing continuous deployment since October 2018. The local Argentinean team has a challenging task of transporting the SSDs to different WCD stations located in hard to reach areas, but has still been able to install $\sim 6$ detectors per working day. As of July 2019, over 350 detectors have been deployed, of which 77 are operational. The SSDs will be equipped with PMTs, and also new electronics will be installed, as soon as these components become available. The deployment of the SSDs should be completed in 2020.

\section{References}

[1] Pierre Auger Collaboration, The Pierre Auger Cosmic Ray Observatory, Nucl. Instrum. Meth. A 798 (2015) 172.

[2] Pierre Auger Collaboration, The Pierre Auger Observatory Upgrade - Preliminary Design Report, arXiv: 1604.03637.

[3] D. Martello for the Pierre Auger Collaboration, The Pierre Auger Observatory Upgrade, in proceedings of ICRC2017, POS ( ICRC2017) 383 (2017).

[4] Pierre Auger Collaboration, Combined fit of spectrum and composition data as measured by the Pierre Auger Observatory, JCAP 04 (2017) 038.

[5] D. Nitz for the Pierre Auger Collaboration, New Electronics for the Surface Detectors of the Pierre Auger Observatory, in proceedings of ICRC2019, POS ( ICRC2019) 370 (2019).

[6] A. M. Botti for the Pierre Auger Collaboration, The AMIGA underground muon detectorperformance and event reconstruction, in proceedings of ICRC2019, POS ( ICRC2019) 202 (2019).

[7] B. Pont for the Pierre Auger Collaboration, A large radio detector at the Pierre Auger Observatory measuring the properties of cosmic rays up to the highest energies, in proceedings of ICRC2019, POS (ICRC2019) 395 (2019).

[8] D. Beznosko, A. Bross, A. Dyshkant, A. Pla-Dalmau, and V. Rykalin, FNAL-NICADD Extruded Scintillator, IEEE Nuclear Science Symposium (2004), FERMILAB-PUB-05-344.

[9] R. Šmída for the Pierre Auger Collaboration, Scintillator detectors of AugerPrime, in proceedings of ICRC2017, POS ( ICRC2017) 390 (2017).

[10] A. Taboada for the Pierre Auger Collaboration, Analysis of Data from Surface Detector Stations of the AugerPrime Upgrade, in proceedings of ICRC2019, POS ( ICRC2019) 434 (2019). 